\title{
Computed Tomography as an Adjunct to Ultrasound in the Diagnosis of Acute Acalculous Cholecystitis
}

\author{
Francis Blankenberg, ${ }^{1}$ Rob Wirth, ${ }^{1}$ R. Brooke Jeffrey Jr., ${ }^{1}$ \\ Robert Mindelzun, ${ }^{1}$ and Isaac Francis, ${ }^{2}$ \\ ${ }^{1}$ Department of Diagnostic Radiology, Stanford University School of Medicine, Stanford, California, USA; \\ and ${ }^{2}$ Department of Diagnostic Radiology, University of Michigan, Ann Arbor, Michigan, USA
}

\begin{abstract}
The sonographic and computed tomographic (CT) findings were reviewed in 17 patients with acute acalculous cholecystitis (AAC) over a 6-year period from 1984 to 1989 . Of the six patients in whom both ultrasound and CT were performed, $\mathrm{CT}$ revealed marked gallbladder (GB) wall abnormalities, including perforation, and pericholecystic fluid collections in five patients not demonstrated by sonography. Of the total group, five patients had GB wall thicknesses of $\leq 3 \mathrm{~mm}$ (normal) at pathologic examination, which demonstrated a spectrum of disease ranging from acute hemorrhagic/necrotizing, to gangrenous acalculous cholecystitis with perforation. Sonography was falsely negative or significantly underestimated the severity of AAC in seven of the 13 patients examined by sonography. CT because of its superior ability to assess pericholecystic inflammation may provide additional diagnostic information even after a thorough sonographic study in cases of AAC.
\end{abstract}

Key words: Acute acalculous cholecystitis, CT Gallbladder wall.

Since the entity of acute acalculous cholecystitis (AAC) was defined by Glenn in 1947, the definitive preoperative diagnosis of this disease by clinical and biochemical data has proven difficult $[1,2]$. Several authors have used sonographic imaging criteria for the diagnosis of AAC. Criteria used by Shuman et al. [3] included gallbladder (GB) wall thickening of $\geq 6 \mathrm{~mm}$ (focal or diffuse), pericholecystic fluid, and a visual impression of GB enlargement, and/or medium level nonshadowing intralu-

Address offprint requests to: Francis Blankenberg, M.D., Department of Diagnostic Radiology, Stanford University School of Medicine, 300 Pasteur Drive, Stanford, CA 94305, USA minal echoes, suggestive of pus in the presence of a sonographic Murphy's sign. Several authors including Mirvis et al. [4-6] have used sonographic criteria of GB wall thickening $\geq 4 \mathrm{~mm}, \mathrm{~GB}$ distention $(\geq 4 \mathrm{~cm}$ maximal transverse diameter or $\geq 8 \mathrm{~cm}$ longitudinally), pericholecystic fluid collections, subserosal edema (halo), and a $<50 \%$ decrease of the transverse or longitudinal GB diameter after intravenous cholecystokinin, which when taken in various combinations is predictive of AAC. In addition, Mirvis et al. suggested that the absence of these findings excluded the diagnosis of AAC, in a group of 60 patients following major trauma [5].

No study has systematically analyzed the relative value of ultrasonography and computed tomographic (CT) examinations in patients with AAC. A retrospective analysis of imaging and pathologic records between the years of 1984 and 1989 of 17 cases of AAC was performed in an unselected group of patients.

\section{Subjects and Methods}

Patients were included from Stanford University Medical Center, Santa Clara Valley Medical Center, San Francisco General Hospital, Palo Alto Veteran's Administration Hospital, and the University of Michigan Medical Center. Sonographic examinations were performed with the use of the Acuson 128 $(\mathrm{N}=6)$ or the Diasonics DRF-400 $(\mathrm{N}=7)$ scanners with 3.5-MHz transducers. CT examinations were performed with several axial $1.0-\mathrm{cm}$ slices through the upper abdomen with the GE $9800 / 8800(\mathrm{~N}=5 / \mathrm{N}=1)$, Toshiba TCT $80 \mathrm{~A}(\mathrm{~N}=2)$, and Picker 1200 SX Siemens Somatome DR $(N=1)$ scanners. Intravenous contrast consisted of 120-150 $\mathrm{ml}$ of $60 \%$ Hypaque and was given in eight of $10 \mathrm{CT}$ examinations.

Of the total group of 17 patients with pathologically proven AAC, nine patients underwent sonographic or CT examination within $24 \mathrm{~h}$ prior to laparotomy, an additional three patients were imaged $72 \mathrm{~h}$ prior to laparotomy, and the remainder between 4 and 6 days prior to laparotomy. Six patients had sonography within 7 days prior to the CT exam (three within 
$72 \mathrm{~h}$ ). There were 12 men and five women with an average age of 63 years, ranging from $27-93$ years. Thirteen patients had suspected acute cholecystitis prior to imaging. Four patients had no clinical suspicion of biliary disease, and exams were performed to rule out an intraabdominal abscess.

Six patients had major surgical procedures, including aortic and mitral valve replacements, gastrojejunostomy, partial small bowel resection with splenectomy, femoral popliteal artery bypass grafting, and redo coronary artery bypass grafting, prior to laparotomy for AAC. Risk factors of these patients included hyperlipidemia, primary amyloidosis, insulin-dependent diabetes mellitus, coronary artery disease, and age $>80$ years. Two patients experienced major blunt trauma, one with severe neurofibromatosis, the other suffered significant small bowel injury. Three patients had diabetes mellitus with or without insulin dependence. Two patients were severe arteriopaths, one with a recent aortoiliac thrombosis, the other had suffered a recent subendocardial myocardial infarction. The remaining four patients had no other risk factors other than age $>80$ years.

\section{Results}

\section{Ultrasound}

Ultrasound was performed in 13 patients, 12 of whom had suspected acute cholecystitis and one with suspected intraabdominal abscess. The sensitivity of sonography was $23 \%$ prospectively, and $46 \%$ retrospectively by Mirvis' criteria with the addition of a sonographic Murphy's sign as a major criterion. Only three patients, all imaged within $72 \mathrm{~h}$ prior to laparotomy, had sonographic findings that were definitely interpreted as AAC prospectively. The findings included GB wall thickening, (i.e., $\geq 5 \mathrm{~mm}$ thick wall, $\geq 5 \mathrm{~cm}$ maximal transverse diameter), subserosal edema, and pericholecystic fluid and sonographic Murphy's sign. Four patients were found to have normal GB wall thicknesses of $\leq 3 \mathrm{~mm}$ at both sonographic and pathologic examination. All (four patients) had mild $\mathrm{GB}$ distension ( $\leq 5 \mathrm{~cm}$ maximal transverse diameter). Only one of these patients had a positive sonographic Murphy's sign, another had an extensive pericholecystic fluid collection thought to be due to ascites, which later proved to be a fundal perforation with a subphrenic abscess. Histology of this group of patients ranged from focal intramural hemorrhage to necrosis with intramural candidiasis.

Three patients with proven AAC were found to have normal GB wall thicknesses of $\leq 3 \mathrm{~mm}$ without GB distension. Laparotomy was performed between 3 and 11 days after imaging. All were found to have acute hemorrhagic or gangrenous cholecystitis, two had markedly thickened GB walls of $\geq 0.8 \mathrm{~cm}$. The third patient had a mildly thickened wall (no measurement given) at laparotomy.
The remaining patients had minimal sonographic findings of mildly thickened walls (4-5 mm), mild GB distension, and subserosal edema $(\mathrm{N}=2)$. Three underwent laparotomy within $72 \mathrm{~h}$ of imaging. The remaining patient underwent laparotomy 6 days after imaging. All had severe $\mathrm{AAC}$ with extensive mucosal hemorrhage and wall thicknesses of $\geq 0.4-0.8 \mathrm{~cm}$. One patient sustained a frank GB perforation.

\section{$C T$}

CT was performed in 10 patients. The clinical indications for CT included suspected acute cholecystitis, small bowel perforation versus biliary obstruction, and intraabdominal abscess. Six patients had sonography within 7 days prior to the CT exam, three within $72 \mathrm{~h}$. CT demonstrated markedly irregular GB wall thickening, streaky soft tissue densities in the pericholecystic fat, as well as pericholecystic fluid either not seen or grossly underestimated by sonography in four cases, as well as a frank fundal perforation. Only one patient had definite findings of $\mathrm{AAC}$ at sonography and at CT retrospectively. CT was performed after an ultrasound examination, as the sonographic findings were minimal in two cases or normal in three cases (see Figs. 1 and 2). All five patients were found to have gangrenous cholecystitis at laparotomy, performed 1 to 4 days after CT examination.

Four patients had CT examination only prior to laparotomy. Three were imaged within $72 \mathrm{~h}$ of laparotomy. One patient was imaged 6 days prior to laparotomy. Two patients of this group had apparently normal GBs at CT examination but had concurrent postoperative ascites. The third patient had a markedly enlarged GB with extensive pericholecystic fluid and edema of the pericholecystic fat and the right perirenal fascia. The fourth patient demonstrated the classic CT findings of $\mathrm{AAC}$, pericholecystic fat soft tissue streaking and massive GB distension and wall thickening $(>1.0 \mathrm{~cm})$. The sensitivity of CT was $50 \%$ prospectively and $70 \%$ retrospectively, with the CT criteria used by Mirvis et al. [6].

\section{Hepatobiliary Scintigraphy}

Six patients underwent hepatobiliary scintigraphy 1 to 6 days prior to laparotomy. Three patients had an absence of GB activity 90 min after injection of the radiopharmaceutical, one had a "rim sign" of activity in the region of the GB fossa. Three patients demonstrated apparently normal $(\mathrm{N}=1)$ to delayed $(\mathrm{N}=2) \mathrm{GB}$ filling within $90 \mathrm{~min}$ without evidence of intraperitoneal leakage of the 

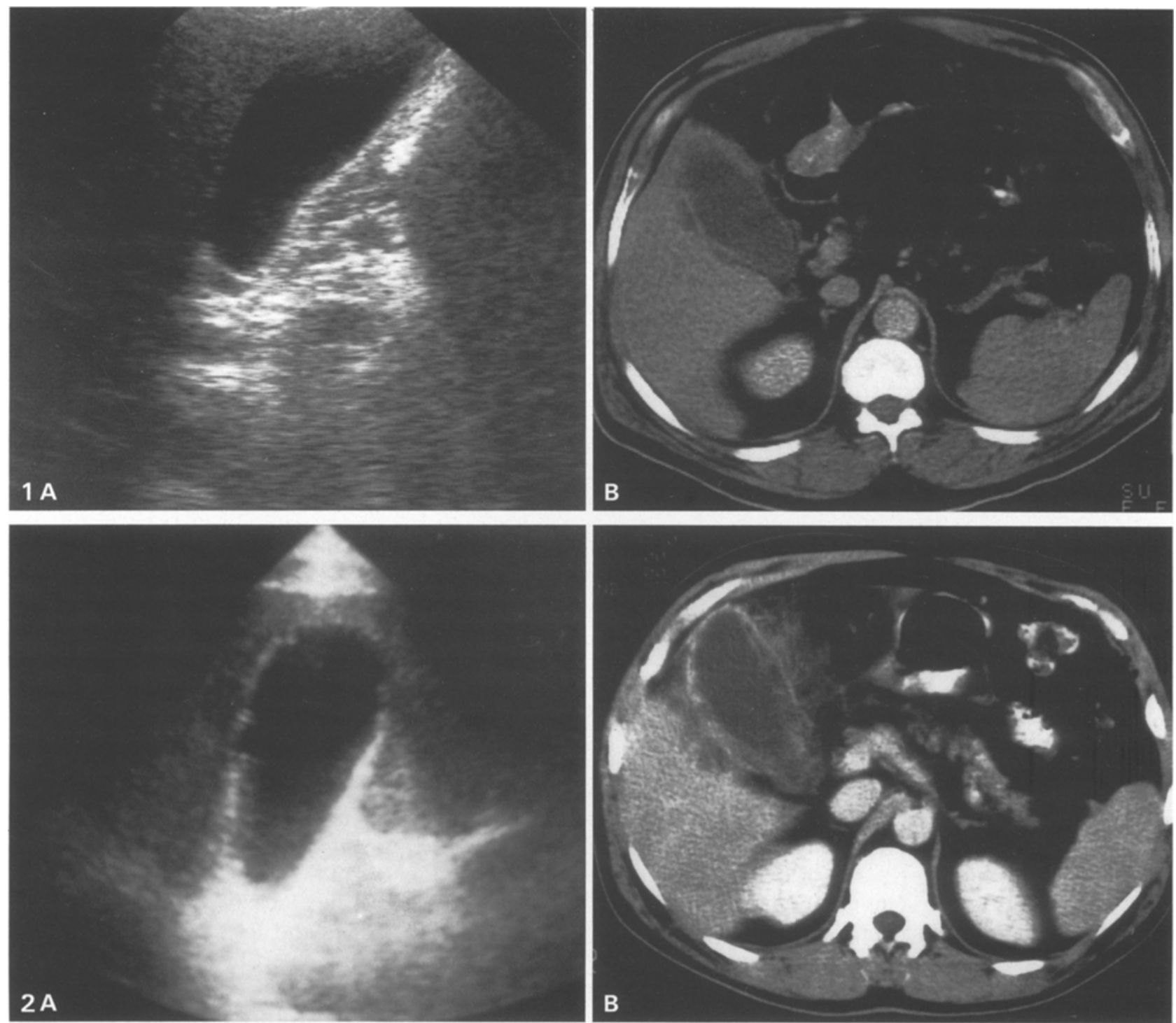

Fig. 1. A 68-year-old diabetic woman presented with right upper quadrant pain for several days. A Ultrasound performed on February 23, 1990. B CT scan performed on March 3, 1990. The ultrasound was considered negative.

Fig. 2. A 38-year-old man presented with right upper quadrant pain and E. coli sepsis. A Ultrasound performed on May 7, 1989. B CT scan performed on May 10, 1989. The ultrasound was considered to show mild wall thickening.

radiopharmaceutical. One patient had a fundal perforation, the other two patients had acute necrotizing cholecystitis.

\section{Discussion}

AAC is usually a difficult clinical diagnosis and is frequently unsuspected in critically ill patients

$[1,2]$. Only half of the patients with AAC will have right upper quadrant tenderness and hyperbilirubinemia. The remainder may present with fever with or without leukocytosis [5]. Several authors have emphasized that only one third of patients with confirmed gangrenous cholecystitis (with or without cholelithiasis) will have a clear sonographic Murphy's sign [7]. Furthermore, diagnostic aspiration of the GB in patients with AAC is also an insensitive technique [8]. Despite these clinical limitations, imaging with both ultrasound and $\mathrm{CT}$ has been felt to be quite sensitive and specific in the diagnosis of AAC $[4-6,9,10]$.

The majority of our patient population $(\mathrm{N}=$ 13) had suspected acute cholecystitis, but did not suffer major trauma as in the series published by Mirvis and Cornwell. Therefore, imaging was undertaken to rule-out "calculous cholecystitis" as 
opposed to AAC. CT was performed after sonography revealed minimal abnormalities or was indeed negative. Perhaps the fact that the current series of patients was "unselected" without a high prior probability of AAC may in part explain the lack of sensitivity of sonography at detecting both the presence and degree of AAC as compared to the findings of Mirvis and Cornwell. Furthermore, Johnson et al. [11] found that up to one third of patients with AAC had normal GB wall thicknesses of $\leq 3 \mathrm{~mm}$ at pathologic examination. Clearly, normal GB wall thickness in no way excludes profound intramural inflammation, hemorrhage, or gangrene, as confirmed by the five patients whose GB wall thicknesses were measured at pathology in our study.

The lack of sensitivity of sonography of our series is further underscored by the seven patients with normal exams [GB wall thickness $\leq 3 \mathrm{~mm}$, no pericholecystic fluid or subserosal edema or intraluminal membranes, or intramural gas or GB distension $(\mathrm{N}=7)$ ] and mildly abnormal exams [GB wall thickness 4-5 mm, GB distension $\leq 5 \mathrm{~cm}$ maximum transverse diameter, subserosal edema, no pericholecystic fluid collection $(\mathrm{N}=4)$ ]. The paucity of sonographic findings may have been partly responsible for the delay in surgical treatment (between 3 and 11 days after imaging). Follow-up CT examinations performed in five of these cases (two within $72 \mathrm{~h}$, two 7 days after sonography) revealed significant pericholecystic fluid collections, edematous pericholecystic fat, and markedly thickened, irregularly enhancing GB walls not demonstrated by ultrasound. There was a significant time delay between sonographic and CT examination in several cases that in part may explain the marked discrepancy between the sonographic and CT findings. However, it is quite disturbing that the sonographic findings in symptomatic patients early in the course of AAC were minimal. Whether CT would provide supplementary data to sonography in all patients with $\mathrm{AAC}$ is unknown, but work by Smathers et al. [9] suggests that CT is quite sensitive (although not specific) for complicated cholecystitis. Our series had two patients in whom only CT was performed (1 and 6 days prior to laparotomy) in which the GB wall thickness and size were normal, but both had ascites with pericholecystic fluid.

Recently, Fig et al. [12] found that hepatobiliary scintigraphy performed with morphine augmentation had a false-positive rate of up to $60 \%$ in severely ill patients. In addition, Raduns et al. [13] found a lack of utility of cholecystokinin sonography in diagnosing $\mathrm{AAC}$ when hospitalized patients without biliary disease were studied, as the majority of patients had subnormal contractility ( $<50 \%$ decrease of length, width after intravenous injections of cholecystokinin). Surprisingly, hepatobiliary scintigraphy demonstrated normal to delayed GB filling in three patients of our series undergoing Hida scans prior to laparotomy, one of which had GB perforation. GB contractility was not assessed with cholecystokinin, although a diseased GB would not be expected to contract normally. Unfortunately, the lack of GB contractility with intravenous cholecystokinin can be a normal finding in up to one fourth of hospitalized patients. The reason for GB filling is unclear, but could imply that functional cystic duct obstruction may not be the primary cause for AAC in these patients.

Profound ischemia may be the primary insult in the development of AAC. GB mucosal ischemia would be worsened by distension, as well as underlying small vessel disease. Varying degrees of ischemic insult to the GB mucosa could partly explain the wide spectrum of wall abnormalities in our population. Experimental work by Howard et al. [14] found that unilateral and bilateral sympathectomy were both effective in drastically reducing the incidence of acute cholecystitis (in a canine model) in an additive fashion. A case report of focal AAC with perforation was described by Warshauer [15]. Certainly cystic duct obstruction was not the cause of AAC in this patient. There was no evidence of vasculitis; however, focal ischemic disease is an alternative explanation for the perforation. Glenn and Becker [16] upon histologic examination of GBs from patients with $\mathrm{AAC}$ felt vascular changes due to endothelial damage, or arterial spasm lead to thrombosis of the blood vessels of the GB. Therefore, thrombosis of GB vessels as opposed to functional cystic duct obstruction in their view was the primary insult leading to AAC.

In Howard's work with a canine model it was necessary to instill $2 \mathrm{~g}$ of sodium dehydrocholate acid and distend the GB prior to cystic duct ligation to produce acute cholecystitis. Simple cystic duct ligation without injection was insufficient to cause acute cholecystitis. These findings may suggest that GB distension (probably the equivalent of ischemia in the canine model) and concentrated bile salts are both necessary for the development AAC. A breakdown of normal mucosal barrier even focally would allow supersaturated toxic bile to come in contact with the muscular GB wall. This event, although it would have catastrophic consequences for the GB, may not cause significant wall thickening early in the disease. If mucosal 
breakdown is a major factor in AAC, then hyperbilirubinemia would not be an unexpected finding. Bacterial involvement may indeed be a secondary phenomenon in AAC, which would explain the insensitivity of bile cultures via operative or percutaneous cholecystostomy. In addition, drainage may be of major benefit in AAC even without bacterial involvement by lessening the degree of chemical insult to the GB wall.

\section{Conclusions}

Sonography may be falsely negative or may significantly underestimate the severity of AAC. In advanced cases of AAC, CT may provide additional information by detecting pericholecystic inflammation and gross GB wall abnormalities even with negative or minimal findings on sonographic exam.

Hepatobiliary scintigraphy may be falsely negative in AAC, suggesting that functional cystic obstruction may not be the primary factor in the development of the disease. Normal GB wall thicknesses of $\leq 3 \mathrm{~mm}$ at sonography and CT, as confirmed pathologically, does not exclude AAC with gangrene or perforation, and underscores the possibility that focal or diffuse ischemic insults to the GB mucosa may be the primary etiology of AAC.

\section{References}

1. DuPriest RW, Khaneja S, Cowley AR. Acute cholecystitis complicating trauma. Ann Surg 1979; 189:84-89

2. Glenn F. Acute acalculous cholecystitis. Ann Surg 1979; $189: 458-465$
3. Shuman WP, et al. Low sensitivity of sonography and cholescintigraphy in acalculous cholecystitis. Am J Radiol 1984; 142:531-534

4. Mirvis S, et al. The diagnosis of acute acalculous : a comparison of sonography, scintigraphy, and CT. Am J Radiol 1986; 147:1171-1175

5. Cornwell E, Rodriquez A, Mirvis SE, Shorr RM. Acute acalculous cholecystitis in critically injured patients. Ann Surg 1989; 210:52-55

6. Mirvis SE, Whitley NO, Miller JW. CT dignosis of acalculous cholecystitis. J Comput Assist Tomogr 1987; 11:83-87

7. Simeone JF. The sonographic diagnosis of acute gangrenous cholecystitis: importance of the Murphy sign. Am I Radiol $1989 ; 152: 289-290$

8. McGahan JP, Lindfors KK. Acute cholecystitis: diagnosis accuracy of percutaneous aspiration of the gall bladder. $R a$ diology 1987; 167:669-671

9. Smathers RL, Lee JK, Heiken JP. Differential of complicated cholecystitis from gall bladder carcinoma by computed tomography. Am I Radiol 1984; 143:255-259

10. Becker CB, Burckhardt B, Terrier F. Ultrasound in postoperative acalculous cholecystitis. Gastrointest Radiol 1986; $11: 47-50$

11. Johnson LB. The importance of early diagnosis of acute acalculous cholecystitis. Surgery 1987; 164:197-203

12. Fig LM, et al. Morphine-augmented hepatobiliary scintigraphy in the severely ill : caution is in order. Radiology 1990; 175:467-473

13. Raduns K, McGahan JP, Beal S. Cholecystokinin sonography: lack of utility in diagnosis of acute acalculous cholecystitis. Radiology 1990; 175:463-466

14. Howard JM, Milford MT, DeBakey ME. The significance of the sympathetic nervous systems in acute cholecystitis. Surgery $1952 ; 251-258$

15. Warshauer D. Focal acute acalculous cholecystitis. Am J Radiol 1987; 149:505-506

16. Glenn F, Becker CB. Acute acalculous cholecystitis - an increasing entity. Ann Surg 1982; 195:131-136

Received: June 14, 1990; accepted: July 18, 1990 